BMJ Open

Diabetes

Research

\& Care

\title{
Validation and feasibility of a postal system for remote monitoring of $\mathrm{HbA1c}$
}

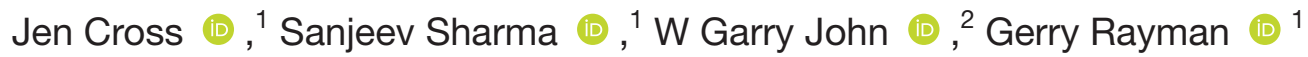

To cite: Cross J, Sharma S, John WG, et al. Validation and feasibility of a postal system for remote monitoring of $\mathrm{HbA} 1 \mathrm{C}$. BMJ Open Diab Res Care 2021;9:e002527. doi:10.1136/ bmjdrc-2021-002527

- Additional supplemental material is published online only. To view, please visit the journal online (http://dx.doi. org/10.1136/bmjdrc-2021002527).

Received 5 August 2021 Accepted 17 October 2021

\section{Check for updates}

(C) Author(s) (or their employer(s)) 2021. Re-use permitted under CC BY-NC. No commercial re-use. See rights and permissions. Published by BMJ.

${ }^{1}$ Deptartment of Diabetes and Endocrinology, Ipswich Hospital NHS Trust, Ipswich, UK ${ }^{2}$ Clinical Biochemistry, Norfolk and Norwich University Hospital NHS Trust, Norwich, UK

Correspondence to Professor Gerry Rayman; gerry.rayman@esneft.nhs.uk

\section{ABSTRACT}

Introduction The COVID-19 pandemic has reduced the accessibility to hemoglobin $\mathrm{A} 1 \mathrm{c}(\mathrm{HbA} 1 \mathrm{c})$ tests required for virtual diabetes clinics. The aim was to develop and validate a user-friendly postal system for remote $\mathrm{HbA} 1 \mathrm{c}$ monitoring.

Research design and methods Validation: A total of 123 capillary blood samples from people with diabetes (PWD) needing face-to-face consultations along with healthy volunteers were measured on a point-of-care (POC) Siemens DCA Vantage Analyzer. Another sample of 5-10 drops was simultaneously collected in a K2EDTA tube (BD Microtainer) and stored for up to 12 days at room temperature for subsequent retesting. Feasibility: During October to December 2020, a total of 286 postal HbA1c kits were sent to PWD prior to their virtual consultation. These contained sample collection guidance, the necessary equipment and a feedback form. As per Packing Instruction 650 regulations, these were posted back to the diabetes center for $\mathrm{HbA} 1 \mathrm{c}$ testing on the POC analyzer. Results There was a strong correlation between the first and the stored sample $\left(R^{2}=0.978\right)$. There was a small clinically insignificant negative bias $-1.53 \mathrm{mmol} / \mathrm{mol}(2$ $\mathrm{SD}=3.10 \mathrm{mmol} / \mathrm{mol}$ ). Bland-Altman plots showed $93 \%$ of results within 2 SD. Of the $87 \%$ of returned kits, only one sample failed to be analyzed. $94 \%$ of PWD who provided feedback were happy to use the postal HbA1c system again.

Conclusions A robust user-friendly postal $\mathrm{HbA1c}$ system has been created and successfully integrated into clinical practice using the existing POC equipment at the diabetes center. It provides accurate $\mathrm{HbA1c}$ results and is an invaluable tool for remote monitoring of $\mathrm{HbA1c}$ in PWDboth during and after the pandemic.

\section{INTRODUCTION}

The COVID-19 pandemic has led to a significant number of National Health Service (NHS) clinics being moved from the traditional face-to-face model to virtual consultations using telemedicine. Unfortunately, the need for social distancing and patient-related hesitation to attend healthcare facilities have made it difficult to obtain a hemoglobin A1c (HbAlc) measurement for use at the virtual consultation. Newer technologies such as flash glucose sensing and continuous glucose monitoring are being increasingly used and provide invaluable data for assessing glycemic control. However, for the majority of people

\section{Significance of this study}

What is already known about this subject?

- Hemoglobin $\mathrm{A} 1 \mathrm{c}(\mathrm{HbA1C})$ measurement is an important tool for monitoring glycemic control in people with diabetes (PWD), but unfortunately during the COVID-19 pandemic access to an up-to-date HbA1c measurement for PWD has been challenging.

What are the new findings?

- We describe an inexpensive, simple to implement and accurate system for at-home blood collection, postage and testing; providing accurate $\mathrm{HbA1C}$ results for virtual consultations.

- The at-home capillary blood collection system was well received by PWD.

- Our system provides clinicians the opportunity to obtain an up-to-date HbA1c from PWD prior to a virtual consultation.

How might these results change the focus of research or clinical practice?

- Our at-home blood collection system enables PWD to independently collect their blood sample in the comfort of their own home and safely return it to the diabetes center for analysis, thus providing clinicians with this important information prior to remote consultation using virtual telemedicine.

with diabetes (PWD), HbA1c remains the critical tool for evaluating glycemic control over a period of 100-120 days.

Remote HbAlc monitoring could potentially provide a solution, and to date a number of blood collection methods have been described, including dried blood spot (DBS) collection, volumetric absorptive microsampling (VAMS) and commercial kits. ${ }^{1-7}$ Unfortunately, each has significant issues preventing widespread clinical us. Some have been reported to have inconsistent accuracy, ${ }^{1}$ while others involve a lengthy timeconsuming process, ${ }^{2-4}$ many are economically non-viable and there is a significant lack of feedback from PWD to support their use in routine diabetes care. ${ }^{5-7}$

For a remote HbAlc blood collection system to be adopted successfully into clinical practice, it needs to provide accurate and 
reproducible results and needs to be economically viable and user friendly for PWD to collect samples and for NHS staff to process these.

The Diabetes Centre at Ipswich Hospital (East Suffolk and North Essex NHS Foundation Trust) employs two point-of-care Siemens DCA Vantage Analyzers (Siemens Healthcare Diagnostics, USA) which provide reliable HbA1c results within $6 \mathrm{~min}$. Use of the analyzers in parallel increases sampling throughput. These analyzers have been in routine use in our diabetes center for over 20 years and are widely available in clinics throughout the UK.

The aim of this article is to describe how we developed an inexpensive, effective system for home collection and postal return of capillary blood samples for remote HbA1c measurement; and to determine the validity of the HbAlc results where the postal system results in a delay in its measurement.

\section{METHODS}

Initial concept testing: handling of capillary samples and accuracy testing following storage up to 12 days

For the postal HbAlc blood collection system to be considered effective, three important criteria had to be fulfilled: first, it was necessary to demonstrate that the samples assessed after a period of storage (due to postal transit) could still be processed by the Siemens DCA Vantage analyzers and analytical quality maintained. Second, the method needed to comply with specific postal regulations and, finally, there needed to be good uptake and acceptance by PWD.

A total of 123 capillary blood samples were collected from both, consenting PWD ( $n=94)$ attending the diabetes center for face-to-face consultations and healthy volunteers $(\mathrm{HV})(\mathrm{n}=29)$. The latter were colleagues from the Ipswich Hospital. One microliter of capillary blood was collected by laboratory staff at the Ipswich Diabetes Centre into the capillary tube supplied with the DCA cartridge and immediately tested on the Siemens DCA Vantage Analyzer (Siemens, UK) as per standard procedures. Thereafter, both PWD and HV were asked to follow a photographic guide and collect approximately 50-100 $\mu \mathrm{L}$ blood ( $~ 5-10$ drops) for retesting after storage at room temperature up to 12 days later. The first 33 samples were collected into CB 300 EDTA tubes (Sarstedt, Germany), and after storage were remixed using a mixer (Coulter, USA) for 6 min. Remixed samples were pipetted (Gilson Pipetman P200, USA) and then dispensed onto the non-absorbent side of Benchkote hydrophobic paper (Whatman, USA) to aid the ease of filling of the DCA capillary tube. Two expected errors on the DCA analyzer are 'High Hb' E107 and 'low Hb' E104; these are known to occasionally occur during routine use but were much more frequent with the 'stored' samples. We speculated that the method we had used did not adequately resuspend the red cells in the separated plasma. Furthermore, as PWD found it difficult to use the Sarstedt tubes which rely on capillary fill we considered alternatives. We turned to K2EDTA BD Microtainer collection tubes (BD, UK) which are filled by scooping capillary droplets. Initial use suggested this was easier for patients, so this was used in subsequent evaluations $(n=90)$. We also abandoned the Coulter remixer in favor of a vortex device (Iswix variable speed vortex, Alpha Laboratories, UK) for 30-60 s at $1400 \mathrm{rpm}$.

This strategy all but eliminated E107 and 'low Hb' E104 errors. On the very rare occasion when a high $\mathrm{Hb}$ E107 error occurred, we found that adding $7 \mu \mathrm{L}$ saline to the stored sample and remixing using the vortex overcame the problem. All stored samples were tested on the same DCA Vantage Analyzer as their first HbA1c test to prevent any interdevice bias. The performance of the DCA Vantage Analyzers was assessed daily using reconstituted control fluids (Siemens DCA, USA) before testing HV or PWD samples.

\section{Temperature validation study}

A further temperature validation study has been conducted to examine the effect of blood sample storage at higher ambient temperature. Seventeen random samples (nine HV and eight PWD) were collected and initially tested as above. The samples were then stored at $29.7^{\circ} \mathrm{C} \pm 0.69^{\circ} \mathrm{C}$ (EL-USB-2-LCD+RH/Temp Data Logger, Lascar Electronics, UK) for 24 hours, and thereafter retested on the same DCA analyzer.

\section{Postal method for HbA1c collection}

The aim was for PWD to collect blood samples independently at home and post them back to the diabetes center for processing.

Three factors were considered to establish a blood sample return system that was reliable and cost-effective:

- Compliance to postal regulations (Packing Instruction 650: the European Agreement concerning the International Carriage of Dangerous Goods by $\operatorname{Road}^{89}$ ) (online supplemental data 1).

- Cost-effectiveness of the postal process (online supplemental data 2).

- Convenience/user-friendly nature of the entire process for PWD.

\section{Patient engagement and feedback on the postal HbA1c kits}

Once the accuracy of the stored samples was demonstrated and postal requirements were confirmed, we evaluated the acceptability of the system in PWD requiring an $\mathrm{HbA1c}$ prior to their virtual consultation. During October to December 2020 a total of 286 kits were sent out. The postal HbAlc kits contained a covering letter explaining why a postal HbAlc was required, a photographic blood collection guide, a packaging guide and a feedback form (online supplemental data 2). The kits also contained the necessary equipment to collect the capillary sample, to package it and send it safely back to the diabetes center according to Packing Instruction 650 regulations. 
Table 1 Mean $( \pm$ SD) HbA1c of samples for both people with diabetes (PWD) $(n=94)$ and healthy volunteers $(H V)(n=29)$ on the day of collection and up to 12 days after sample collection

\begin{tabular}{lllll}
\hline $\begin{array}{l}\text { HbA1c (day of } \\
\text { collection) }\end{array}$ & Total samples $(\mathbf{n})$ & Mean $( \pm$ SD) & $\begin{array}{l}\text { HbA1c (at second analysis } \\
\text { up to 12 days) }\end{array}$ & Mean (士SD) \\
\hline PWD & 94 & $65 \mathrm{mmol} / \mathrm{mol} \pm 15.46$ & 94 & $63 \mathrm{mmol} / \mathrm{mol} \pm 15.10$ \\
HV & 29 & $33 \mathrm{mmol} / \mathrm{mol} \pm 4.65$ & 29 & $33 \mathrm{mmol} / \mathrm{mol} \pm 4.45$ \\
\hline
\end{tabular}

$\mathrm{P} \geq 0.05$ : not significant.

HbA1c, glycosylated hemoglobin A1c.

Feedback forms were included with each postal HbA1c kit. All feedback was optional and was related to the use of the of K2EDTA BD Microtainer bottles (BD).

\section{Statistical analysis}

Statistical analysis was performed using SPSS V.22 (IBM SPSS Statistics for Windows) and StatsDirect V.3 (StatsDirect, Cheshire, UK). HbAlc as quantitative variables is expressed as mean+SD. The accuracy between samples for both PWD and HV on the day of collection and up to 12 days after sample collection was estimated using first paired samples t-test for mean and then correlation was tested using the Pearson correlation method (significant was defined as $\mathrm{p}<0.05$ ). To further test the agreement between both sets of sample, we used the Bland-Altman limits of agreement (BA LoA) method using $\mathrm{X}$-axis as mean of both HbAlc sets and Y-axis as the difference between both measurements.

\section{RESULTS}

\section{Stability results}

Table 1 depicts the mean $( \pm \mathrm{SD}) \mathrm{HbA1c}$ on the day of collection and up to 12 days later.

The mean $( \pm \mathrm{SD}) \mathrm{HbA1c}$ for PWD on day of collection was $65 \mathrm{mmol} / \mathrm{mol}(8.1 \%)$ and $63 \mathrm{mmol} / \mathrm{mol}(7.9 \%)$ when retested up to 12 days later with no significance noted $(p>0.05)$. Similarly, for HV, no significance was noted on day of collection $(33 \mathrm{mmol} / \mathrm{mol} 5.4 \%)$ when compared with the retest $(33 \mathrm{mmol} / \mathrm{mol} 5.4 \%)$; $>0.05$. Intervals between initial $\mathrm{HbA} 1 \mathrm{c}$ testing and stored sample HbAlc testing are shown in table 2.

Figure 1 shows the Pearson correlation between the first and the stored HbA1c samples. A strong correlation

Table 2 Total number of capillary samples tested for stability from healthy volunteers and people with diabetes $(n=123)$. The storage intervals used before the second $\mathrm{HbA} 1 \mathrm{c}$ test are shown

\begin{tabular}{lc}
\hline $\begin{array}{l}\text { Number of days between sample } \\
\text { collection and second HbA1c test }\end{array}$ & Samples (n) \\
\hline $1-2$ & 10 \\
$4-7$ & 97 \\
$10-12$ & 16 \\
Grand total & 123 \\
\hline
\end{tabular}

$\mathrm{HbA1c}$, glycosylated hemoglobin A1c. coefficient of $\mathrm{R}^{2}=0.975 ; \mathrm{p}<0.001$ was observed between both samples. This suggests that both sample sets had a significant positive relationship with one another but this may not necessarily depict agreement.

To test the latter, we used the BA LoA method as shown in figure 2. The LoA for the BA plots were set at mean $+1.96 \mathrm{SD}$ and mean $-1.96 \mathrm{SD}$. It shows that there was a significant agreement between both sets of results for both PWD and $\mathrm{HV}$ and $93 \%$ of results within $1.96 \mathrm{SD}$ of the line of bias.

Temperature validation studies involving 17 random samples (nine $\mathrm{HV}$ and eight $\mathrm{PWD}$ ) which were stored at a higher temperature $\left(29.7^{\circ} \mathrm{C} \pm 0.69^{\circ} \mathrm{C}\right.$ for 24 hours $)$ and then retested did not adversely affect the accuracy of the methodology. The mean $( \pm \mathrm{SD}) \mathrm{HbAlc}$ on the day of collection was $47 \mathrm{mmol} \pm 15.87$ as compared with $45.7 \mathrm{mmol} \pm 15.69$ when retested 24 hours later (paired t-test: $\mathrm{p}=0.021$ ). Furthermore, there was a strong positive correlation between the first samples and the stored samples $\left(\mathrm{R}^{2}=0.98\right)$ (online supplemental data 5).

Based on the statistical derivations above, we infer that in both PWD and HV, even when the HbAlc was tested

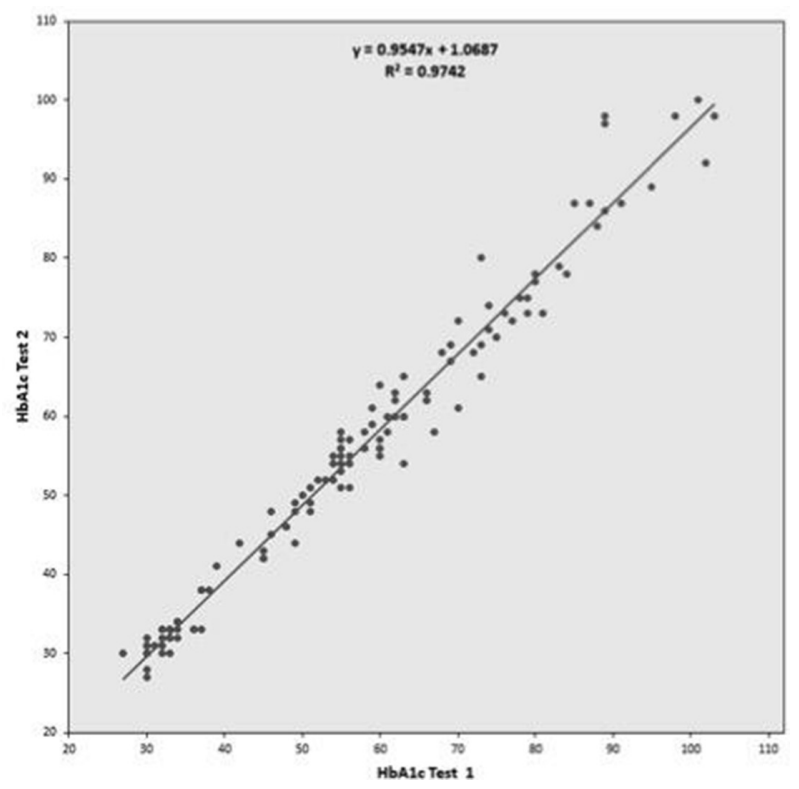

Figure 1 Pearson correlation coefficient for both sample sets of blood. HbA1c test 1 on X-axis indicates samples taken on day of collection and $\mathrm{HbA} 1 \mathrm{c}$ on $\mathrm{Y}$-axis indicates retest results up to 12 days later. $\mathrm{HbA1c}$, glycosylated hemoglobin A1c. 


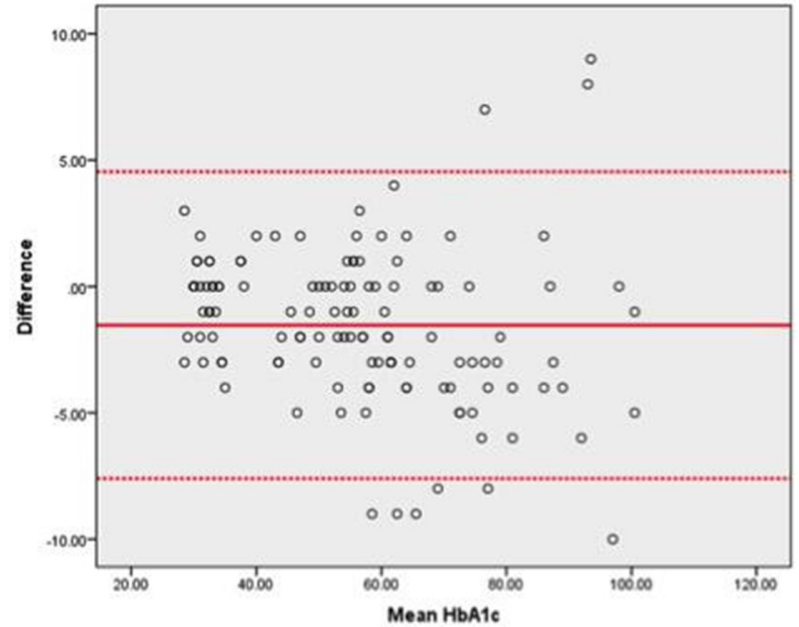

Figure 2 Bland-Altman plot with limits of agreement (LoA) for capillary blood sample HbA1c. The upper LoA has been set at mean+1.96 SD and lower LoA set at mean -1.96 SD. $\mathrm{HbA1c}$, glycosylated hemoglobin A1c.

using the postal system and in different time frames of up to 12 days, there was significant correlation and agreement between both sets of results.

\section{Compliance-return rates}

During October to December 2020, a total of 286 kits were sent out to PWD requiring an HbAlc prior to their virtual consultation (age range 9-86 years, mean age 51 years, table 3 ).

Mean HbA1c for the returned kits was $67 \mathrm{mmol} / \mathrm{mol}$ (8.3\%) (range $31-128 \mathrm{mmol} / \mathrm{mol} ; 5.0 \%-13.9 \%$ ). Nine returned samples were $>126 \mathrm{mmol} / \mathrm{mol}(>14.0 \%)$ and 29 samples were above $97 \mathrm{mmol} / \mathrm{mol}(11.0 \%)$.

Of the 210 returned kits $(73 \%)$, there was only one sample that failed to be analyzed. There were four samples that produced an error but were successfully retested as described in the Methods section. All four errors were expected errors of E107 (high Hb) (n=2) or E104 (low Hb) (n=2).

Of those who did not provide a sample, there were several reasons for not doing so, including no need as already had a recent HbAlc, being out of the country, and deferring their appointment. There were also some very frequent non-attenders who were unlikely to engage. If these were not considered the return rate was $87 \%$.

Table 3 Demographics of people with diabetes (PWD) using the postal $\mathrm{HbA} 1 \mathrm{c}$, October to December 2020

\begin{tabular}{lrlll}
\hline & & \multicolumn{3}{l}{ Age (years) } \\
\cline { 3 - 5 } & $\mathbf{n}$ & Mean & Minimum & Maximum \\
\hline Women & 102 & 54 & 19 & 86 \\
Men & 175 & 52 & 19 & 8 \\
Children (9-18) & 9 & 15 & 9 & 18 \\
Total PWD & 286 & 51 & 9 & 86 \\
\hline
\end{tabular}

$\mathrm{HbA1c}$, glycosylated hemoglobin A1c.

\section{Feedback}

Feedback was received from 84 of the 210 who returned a sample $(40 \%)$. The postal HbA1c was well received with $94 \%$ agreeing that they would use it again (online supplemental data 3).

As expected, collecting the capillary sample proved to be the main problem but although $17 \%$ found it very difficult (online supplemental data 3), approximately half of these would still use the system again.

Notably, some feedback mentioned that the addition of an online video guide would be beneficial to aid sample collection.

\section{Cost}

The cost of the postal HbA1c kit equipment, postage and processing was approximately £2.63 per person, which rose to $£ 3.32$ per person when factoring in non-returned kits (online supplemental data 4).

\section{DISCUSSION}

The COVID-19 pandemic has made it challenging to obtain an up-to-date HbAlc for PWD prior to their diabetes consultations. At the time of designing our system, a reliable, inexpensive and easily implemented method which was acceptable for PWD to collect their capillary blood samples at home and gave accurate results was lacking. ${ }^{1-7}$

DBS collection devices have been considered a possible solution. However, in practice they require multiple, time-consuming processing steps in addition to a final calibration to obtain an HbAlc value. ${ }^{1-467}$ The storage, processing and calibration steps are not standardized, despite many efforts to achieve this and there are conflicting reports of their accuracy. ${ }^{1}$ A universal calibration formula for DBS was proposed by Affan et al ${ }^{1}$; however, Mastronardi et al discovered that this correction formula was unfortunately not applicable to their study DBS results.

Despite only requiring a small amount of blood, DBS may not be an easier alternative to whole capillary blood collection for PWD, as insufficient sample rates of up to $15 \%$ have been reported. ${ }^{7}$ Although Fokkema et a $l^{3}$ produced excellent PWD feedback and accurate results from PWD using DBS, this has not been replicated in other studies. One such study reported home-collected samples were significantly different from the result of the laboratory-collected samples (Hall et al). ${ }^{4}$

A review of DBS studies has shown that HbAlc can only be accurately measured within a narrow range ${ }^{1}$ and is thus not suitable for those with poorer control.

VAMS technology is an alternative blood collection method, but unfortunately the processing and calibration steps are still lengthy and complicated. Although there are some data on the use of VAMS over a range of HbAlc levels, ${ }^{2}$ the device and additional equipment required to process the sample are still expensive, limiting its clinical use. $^{5}$ 
The postal HbA1c system that we have devised solves many of these issues: it is standardized, requires minimal processing, is relatively inexpensive and the HbAlc result is available directly from the Siemens DCA that is locally used and already in place. Our postal HbAlc was shown to be accurate, maintained sample stability for up to 12 days and produced clinically acceptable results. The sample stability time frame allows for any postal delays that may occur over holiday periods and indeed occurred during the pandemic.

Other studies have shown that HbAlc measurements are stable using a Siemens DCA analyzer in samples stored for up to 14 days and up to 21 days using affinity chromatography at room temperature, ${ }^{10} 11$ thus mirroring our results. It has been suggested that sample stability may reduce at temperatures over $30^{\circ} \mathrm{C}^{10}$; however, in the UK, room temperatures seldom exceed this, and of note our accuracy study was undertaken during the summer months in non-air-conditioned rooms. This is further supported by our temperature validation data which demonstrated that an increase in temperature, which may occur during postage, did not significantly affect the stability of the results. A recent paper by Beck $e t a l^{12}$ has also shown that an increase in temperature during postage of samples (up to $40.6^{\circ} \mathrm{C}$ ) did not adversely affect the stability of the results. ${ }^{12}$

The return rate of $73 \%$ was acceptable and could be improved if the referral process identified those in whom a home measurement is appropriate. Since completion of this study, and between January and March 2021, the return rate for 245 kits has improved to $80 \%$.

Although only $40 \%$ provided feedback, it was optional. Also, the study was done during the COVID-19 lockdown period and could have affected the feedback response rates; however, $94 \%$ of those responding were willing to use the system again.

As expected, PWD found physical collection of blood a challenge with $17 \%$ reporting finding this difficult; despite this the majority were willing to use the kits again. Difficulty in sample collection has also been encountered in DBS studies with $33.3 \%$ finding it difficult to apply blood to the DBS device. ${ }^{4}$

To make the sample collection easier, we have subsequently reduced the amount of blood required from 10 to 5 drops (from 100 to $50 \mu \mathrm{L}$ ). Sample collection may also be improved by the laboratory staff at diabetes center demonstrating the method when the PWD attends for phlebotomy for their face-to-face appointment and also by access to an online video which we are producing.

The evaluation involved a cohort of PWD with a wide range of HbA1c levels (31-128 mmol/mol, HbAlc 5.0\%$13.9 \%)$. The simplicity of this system has facilitated its easy integration into the activity at the diabetes center's minilaboratory and although this study was confined to patients attending a diabetes center it could be easily adopted for use in other diabetes care settings. The equipment required for processing is minimal, user friendly, requires little training and is cost-effective. Health economics has not been calculated for other home collection systems, ${ }^{4}$ whereas we have shown that the economic impact of this project is minimal with an estimated cost of $£ 3.32$ per person (online supplemental data 4). This cost includes non-returned kits, the number of which could be reduced by more stringent PWD selection, potentially bringing the cost to between $£ 2.50$ and $£ 3.00$ per person.

Since designing and testing our methodology a paper has been published which assessed two capillary blood collection kits for the measurement of HbAlc in the USA. ${ }^{12}$ Similar to our methodology, the kits were well received by PWD and both kits showed accurate, reproducible results up to 10 days, strengthening our findings. However, the blood samples were collected in clinic and so return rates and patient engagement in a non-controlled environment were not assessed, as were in our paper.

There are a number of limitations to consider for our system. Only the Siemens DCA Vantage Analyzer was used since it is already available in our laboratory; however, we see no reasons why similar results would not be obtained on other analyzers. A further limitation is the evaluation being only undertaken at one center. Again, we see no reasons why other centers could not replicate the results and obtain similar patient engagement. Finally, we have only tested samples stored for up to 12 days. Significant postal delays and laboratory closures during the weekends could delay testing to beyond this time, although this was not the case with any of our samples nor during the recent Christmas and the New Year period.

\section{CONCLUSIONS}

In conclusion, we describe an inexpensive, simple to implement and accurate method for obtaining HbAlc results for remote clinics which has good patient acceptance, and overcomes the many challenges that have hampered DBS and VAMS blood collection. We believe that in addition to necessary face-to-face consultations, virtual consultations supported by remote HbAlc testing such as described will be a significant advance in diabetes care.

Acknowledgements The authors are very grateful to all study participants and colleagues at the Diabetes Centre at Ipswich Hospital. The authors would also like to thank Dr Aida Rajic of the University of Suffolk for their kind donation of equipment and Cogent Technology for providing the temperature monitoring equipment.

Contributors All authors were involved in the writing of the manuscript. JC planned and completed the laboratory work for the validation studies, designed the postal HbA1c system, collected the data and integrated the postal HbA1c system into the diabetes center clinics. SS completed the statistical testing of the data and had an editorial role for the manuscript. WGJ provided technical advice. GR is guarantor for the article, conceived the concept of the postal HbA1C system, and provided technical advice for the validation studies and support for the manuscript.

Funding The authors have not declared a specific grant for this research from any funding agency in the public, commercial or not-for-profit sectors.

Competing interests None declared.

Patient consent for publication Not applicable.

Ethics approval This study was designed and carried out during the peak of COVID-19 pandemic activity in the UK. During this period, the urgent necessity for an alternate method of remote glycemic monitoring was considered to be of 
utmost clinical importance. Hence, urgent ethical considerations for this study were reviewed and ratified by the following institutional board: Diabetes Trials Unit at Ipswich Hospital and supported by R\&D at Ipswich Hospital, East Suffolk and North Essex NHS Foundation Trust, so that it could be adopted early in clinical practice. In view of the same clinical reasons, it was deemed that submission to formal NRES Ethics Committee adjudication was not necessary due to COVID-19-related delays. All PWD and healthy HV gave informed consent before taking part.

Provenance and peer review Not commissioned; externally peer reviewed.

Data availability statement All data relevant to the study are included in the article or uploaded as supplemental information.

Supplemental material This content has been supplied by the author(s). It has not been vetted by BMJ Publishing Group Limited (BMJ) and may not have been peer-reviewed. Any opinions or recommendations discussed are solely those of the author(s) and are not endorsed by BMJ. BMJ disclaims all liability and responsibility arising from any reliance placed on the content. Where the content includes any translated material, BMJ does not warrant the accuracy and reliability of the translations (including but not limited to local regulations, clinical guidelines, terminology, drug names and drug dosages), and is not responsible for any error and/or omissions arising from translation and adaptation or otherwise.

Open access This is an open access article distributed in accordance with the Creative Commons Attribution Non Commercial (CC BY-NC 4.0) license, which permits others to distribute, remix, adapt, build upon this work non-commercially, and license their derivative works on different terms, provided the original work is properly cited, appropriate credit is given, any changes made indicated, and the use is non-commercial. See: http://creativecommons.org/licenses/by-nc/4.0/.

ORCID iDs

Jen Cross http://orcid.org/0000-0002-3351-8004

Sanjeev Sharma http://orcid.org/0000-0001-6386-2052

W Garry John http://orcid.org/0000-0001-9681-3679

Gerry Rayman http://orcid.org/0000-0003-3331-7015

\section{REFERENCES}

1 Affan ET, Praveen D, Chow CK, et al. Comparability of HbA1c and lipids measured with dried blood spot versus venous samples: a systematic review and meta-analysis. BMC Clin Pathol 2014;14:1472-6890.

2 Verougstraete N, Lapauw B, Van Aken S, et al. Volumetric absorptive microsampling at home as an alternative tool for the monitoring of HbA1c in diabetes patients. Clin Chem Lab Med 2017;55:462-9.

3 Fokkema MR, Bakker AJ, de Boer F, et al. HbA1c measurements from dried blood spots: validation and patient satisfaction. Clin Chem Lab Med 2009;47:1259-64.

4 Hall JM, Fowler CF, Barrett F, et al. $\mathrm{HbA}_{1 \mathrm{c}}$ determination from HemaSpot ${ }^{\mathrm{TM}}$ blood collection devices: comparison of home prepared dried blood spots with standard venous blood analysis. Diabet Med 2020;37:1463-70.

5 Rudge J. Using remote blood collection with VAMS to accurately measure HbA1c in diabetes. Neoteryx 2020, 2020. Available: https:// www.neoteryx.com/hubfs/Content/Technical\%20Briefs/HbA1cTech-Review-v5.pdf [Accessed Mar 2021].

6 Mastronardi CA, Whittle B, Tunningley R, et al. The use of dried blood spot sampling for the measurement of HbA1c: a crosssectional study. BMC Clin Pathol 2015;15:13.

7 Jones TG, Warber KD, Roberts BD. Analysis of hemoglobin A1c from dried blood spot samples with the Tina-quantR II immunoturbidimetric method. J Diabetes Sci Technol 2010;4:244-9.

8 Department of Transport. Guidance: packaging and transport requirements for patient samples-UN3373. Gov.uk 2020, 2020 Available: https://www.gov.uk/government/publications/packagingand-transport-requirements-for-patient-samples-un3373/packagingand-transport-requirements-for-patient-samples-un3373 [Accessed Mar 2021]

9 Royal Mail. Prohibited and restricted items 2019, 2019. Available: https://personal.help.royalmail.com/app/answers/detail/a_id/96/kw/ prohibited\%20items [Accessed Mar 2021].

10 Rohlfing CL, Hanson S, Tennill AL, et al. Effects of whole blood storage on hemoglobin A1c measurements with five current assay methods. Diabetes Technol Ther 2012;14:271-5.

11 Little RR, England JD, Wiedmeyer HM, et al. Glycosylated hemoglobin measured by affinity chromatography: microsample collection and room-temperature storage. Clin Chem 1983;29:1080-2.

12 Beck RW, Bocchino LE, Lum JW, et al. An evaluation of two capillary sample collection kits for laboratory measurement of $\mathrm{HbA} 1 \mathrm{c}$. Diabetes Technol Ther 2021;23:537-45. 